\title{
A COMPLETE CLASSIFICATION OF FINITE $p$-GROUPS ALL OF WHOSE NONCYCLIC SUBGROUPS ARE NORMAL
}

\author{
ZDRAVKa BoŽIKOV AND Zvonimir JANKO \\ University of Split, Croatia and University of Heidelberg, Germany
}

\begin{abstract}
We give a complete classification of finite $p$-groups all of whose noncyclic subgroups are normal, which solves a problem stated by Berkovich.
\end{abstract}

\section{INTRODUCTION AND PRELIMINARY RESULTS}

We consider here only finite $p$-groups and our notation is standard. If in a $p$-group all cyclic subgroups are normal, then all subgroups are normal and we call such a group Dedekindian. A Dedekindian $p$-group $G$ is either abelian or a Hamiltonian 2-group, i.e., $G=Q \times A$, where $Q \cong Q_{8}$ is the quaternion group and $A$ is elementary abelian (Proposition 1.2). Here we study nonDedekindian $p$-groups all of whose noncyclic subgroups are normal. Such groups have been considered by D.S. Passman [6, Proposition 2.9], but he omitted 2-groups of order $\leq 2^{7}$. In fact here lies the main difficulty. In this paper we refine, improve and extend the arguments of Passman so that all $p$-groups will be included. It turns out that we get in addition five exceptional 2-groups: one group of order $2^{6}$, three groups of order $2^{5}$ and one group of order $2^{4}$. More precisely, we prove the following result which gives a complete classification of the title groups.

Theorem 1.1. Let $G$ be a finite non-Dedekindian p-group all of whose noncyclic subgroups are normal. Then $G$ is one of the following groups.

(i) $G$ is metacyclic minimal nonabelian and $G$ is not isomorphic to $Q_{8}$.

2000 Mathematics Subject Classification. 20D15.

Key words and phrases. Dedekindian p-groups, Hamiltonian 2-groups, minimal nonabelian p-groups, central products. 
(ii) $G=G_{0} * Z$, the central product of a nonabelian group $G_{0}$ of order $p^{3}$ with a cyclic group $Z$, where $G_{0} \cap Z=Z\left(G_{0}\right)$ and if $p=2$, then $|Z|>2$.

(iii) $p=2$ and $G=Q \times Z$ where $Q \cong Q_{8}$ and $Z$ is cyclic of order $>2$.

(iv) $G$ is a group of order $3^{4}$ and maximal class with $\Omega_{1}(G)=G^{\prime} \cong E_{9}$.

(v) $G=\left\langle a, b \mid a^{8}=b^{8}=1, a^{b}=a^{-1}, a^{4}=b^{4}\right\rangle$, where $|G|=2^{5}, G^{\prime} \cong C_{4}$, $Z(G) \cong C_{4}, G^{\prime} \cap Z(G) \cong C_{2}$ and $\Omega_{2}(G)$ is abelian of type $(4,2)$.

(vi) $G \cong Q_{16}$, the generalized quaternion group of order $2^{4}$.

(vii) $G=D_{8} * Q_{8}$, an extraspecial 2-group of order $2^{5}$ and type " - ".

(viii) $G=\left\langle a, b, c \mid a^{4}=b^{4}=[a, b]=1, c^{2}=a^{2}, a^{c}=a b^{2}, b^{c}=b a^{2}\right\rangle$, where $G$ is the minimal non-metacyclic group of order $2^{5}, G$ is a special 2-group with $G^{\prime}=\Omega_{1}(G) \cong E_{4}$.

(ix)

$$
\begin{gathered}
G=\langle a, b, c, d| a^{4}=b^{4}=[a, b]=1, c^{2}=a^{2} b^{2}, a^{c}=a^{-1}, b^{c}=a^{2} b^{-1}, \\
\left.d^{2}=a^{2}, a^{d}=a^{-1} b^{2}, b^{d}=b^{-1},[c, d]=1\right\rangle,
\end{gathered}
$$

where $G$ is a special 2 -group of order $2^{6}$ with $G^{\prime}=\Omega_{1}(G) \cong E_{4}$ in which every maximal subgroup is isomorphic to the minimal non-metacyclic group of order $2^{5}$ (from (viii)).

Conversely, all the above groups satisfy the assumptions of the theorem.

In the proof of our theorem we use the following known results.

Proposition 1.2 (Huppert [3, III.7.12]). Let $G$ be a p-group all of whose subgroups are normal in $G$. Then $G$ is either abelian or a Hamiltonian 2group, i.e., $G=Q \times A$, where $Q \cong Q_{8}$ is the quaternion group and $A$ is elementary abelian.

Proposition 1.3 (L. Rédei, see [2, Lemma 3.1, 3.2]). A p-group $G$ is minimal nonabelian if and only if $d(G)=2$ (minimal number of generators of $G$ is 2) and $\left|G^{\prime}\right|=p$. In that case $\Phi(G)=Z(G)$. A p-group $G$ is metacyclic and minimal nonabelian if and only if $G$ is minimal nonabelian and $\left|\Omega_{1}(G)\right| \leq$ $p^{2}$ in which case either $G \cong Q_{8}$ or

$$
G=\left\langle a, b \mid a^{p^{m}}=b^{p^{n}}=1, a^{b}=a^{1+p^{m-1}}, m \geq 2, n \geq 1\right\rangle .
$$

Proposition 1.4 (see [4, Proposition 1.6]). Let $G$ be a nonabelian 2group of order $\geq 2^{4}$ with $\left|\Omega_{2}(G)\right| \leq 2^{3}$. Then either

$$
\begin{aligned}
& G=\left\langle a, t \mid a^{2^{n}}=t^{2}=1, a^{t}=a^{1+2^{n-1}}, n \geq 3\right\rangle=M_{2^{n+1}} \text { or } \\
& G=\left\langle a, b \mid a^{2^{m-2}}=b^{8}=1, a^{2^{m-3}}=b^{4}, m \geq 5, a^{b}=a^{-1}\right\rangle
\end{aligned}
$$

of order $2^{m}$.

Proposition 1.5. Let $A$ be an abelian maximal subgroup in a nonabelian p-group $G$ and let $g \in G-A$. Then the mapping $x \rightarrow x^{g} x^{-1}(x \in A)$ is a 
homomorphism from $A$ onto $G^{\prime}$ with kernel $Z(G)$. This gives $A / Z(G) \cong G^{\prime}$ and $|G|=p|Z(G)|\left|G^{\prime}\right|$.

Proposition 1.6 (see [4, Proposition 1.10]). Let $G$ be a 2-group of order $\geq 2^{5}$ such that $\Omega_{2}(G)=\langle a, b\rangle \times\langle u\rangle$, where $\langle a, b\rangle=Q \cong Q_{8}$ and $u$ is an involution. Then $G$ is a uniquely determined group of order $2^{5}$. Set $\langle z\rangle=$ $Z(Q)$ so that $a^{2}=b^{2}=z$. There is an element $y$ of order 8 in $G-\Omega_{2}(G)$ such that $y^{2}=u a, u^{y}=u z, a^{y}=a^{-1}$, and $b^{y}=b u$.

Proposition 1.7. (Passman) Let $G$ be a p-group all of whose noncyclic subgroups are normal. Let $H$ be any non-normal subgroup in $G$. Then $H$ is a maximal cyclic subgroup, $\left|G: N_{G}(H)\right|=p$ and $N_{G}(H) / H$ is either cyclic or $p=2$ and $N_{G}(H) / H \cong Q_{8}$ is quaternion.

Proof. By our assumption, $H$ is cyclic. Assume that $H$ is not maximal cyclic and let $K>H$ be a maximal cyclic subgroup containing $H$. Let $L>K$ be a subgroup of $G$ such that $|L: K|=p$. Since $L$ is noncyclic, we have $d(L)=2$ and $L$ is normal in $G$. Thus $|L: \Phi(L)|=p^{2}, \Phi(L)<K$ and $\Phi(L)=\Phi(K)$. But $\Phi(L)$ is normal in $G$ and $H \leq \Phi(L)$ and so $H$ is normal in $G$, a contradiction. We have proved that $H$ is a maximal cyclic subgroup in $G$.

Set $N=N_{G}(H)$ so that $\{1\} \neq N / H$ is Dedekindian. By Proposition 1.2, $N / H$ is either abelian or Hamiltonian. If $N / H$ has two distinct subgroups $N_{1} / H$ and $N_{2} / H$ of order $p$, then $N_{1}$ and $N_{2}$ are normal in $G$ and $N_{1} \cap N_{2}=H$ so that $H$ is normal in $G$, a contradiction. Thus $N / H$ has only one subgroup of order $p$ and so $N / H$ is either cyclic or $p=2$ and $N / H \cong Q_{8}$.

Set $M / H=\Omega_{1}(N / H)$ so that $|M: H|=p$ and $M$ is normal in $G$. Let $H_{0}$ be the subgroup of $H$ with $\left|H / H_{0}\right|=p$. By the first paragraph of the proof, $H_{0}$ is normal in $G$ and since $M$ is noncyclic, $M / H_{0} \cong E_{p^{2}}$. We act with $G / N$ on the set of $p$ non-normal subgroups of order $p$ of $G / H_{0}$ which are contained in $M / H_{0}$, where $H / H_{0}$ is one of them. This forces $|G: N|=p$ and we are done.

Proposition 1.8 ([6]). Suppose that $G$ is a 2-group all of whose cyclic subgroups of order $\leq 4$ are normal in $G$. Then either $\Omega_{2}(G) \leq Z(G)$ or $G$ is Hamiltonian.

Proof. Set $F=\Omega_{2}(G)$ so that $\exp (F) \leq 4$ and suppose that $F \not \leq Z(G)$ which implies $\exp (F)=4$. The subgroup $F$ is Dedekindian and $\Omega_{1}(F) \leq$ $Z(G)$. Since $F \not \leq Z(G)$, there is an element $v$ of order 4 in $F$ and $g \in G$ such that $v^{g}=v^{-1}=v z$, where $v^{2}=z$. Assume that $o(g) \geq 8$. Let $\langle y\rangle$ be the cyclic subgroup of order 4 in $\langle g\rangle$ so that $[v, y]=1$ and $\langle v\rangle \cap\langle y\rangle \leq\langle z\rangle$. We have $(v y)^{g}=v^{-1} y=(v y) z$. If $\langle v\rangle \cap\langle y\rangle=\langle z\rangle$, then $v y$ is a noncentral involution, a contradiction. If $\langle v\rangle \cap\langle y\rangle=\{1\}$, then $o(v y)=4$ and $g$ does not normalize $\langle v y\rangle$. Hence $o(g)=4$ and so $g \in F$ which implies that $F$ is Hamiltonian. 
Assume that $F \neq G$ and let $l$ be an element of order 8 in $G-F$. Then $\langle l\rangle \cap F=\left\langle l^{2}\right\rangle$ and if $u=l^{4}$, then $\langle u\rangle=F^{\prime}=\Phi(F)$. Since $Z(F)=\Omega_{1}(F)$, there is an element $f$ of order 4 in $F$ such that $\left[l^{2}, f\right]=u$ and $f^{2}=u$ so that $\left\langle l^{2}, f\right\rangle \cong Q_{8}$. But $l$ must normalize $\langle f\rangle$ and $l^{2}$ inverts $\langle f\rangle$, a contradiction. We have proved that $G=F$ is Hamiltonian.

Proposition 1.9 (Janko [5, Theorem 2.6]). Let $G$ be a nonabelian 2group all of whose minimal nonabelian subgroups are isomorphic to $\mathrm{H}_{2}=$ $\left\langle a, b \mid a^{4}=b^{4}=1, a^{b}=a^{-1}\right\rangle$. Assume that $\exp (G)=4$ and $\Omega_{1}(G) \cong E_{4}$. Then $G$ is one of the following groups.

(a) $G \cong H_{2}$.

(b) $G$ is the minimal non-metacyclic group of order $2^{5}$ :

$$
G=\left\langle a, b, c \mid a^{4}=b^{4}=[a, b]=1, c^{2}=a^{2}, a^{c}=a b^{2}, b^{c}=b a^{2}\right\rangle .
$$

(c)

$$
\begin{gathered}
G=\langle a, b, c, d| a^{4}=b^{4}=[a, b]=1, c^{2}=a^{2} b^{2}, a^{c}=a^{-1}, b^{c}=a^{2} b^{-1}, \\
\left.d^{2}=a^{2}, a^{d}=a^{-1} b^{2}, b^{d}=b^{-1},[c, d]=1\right\rangle,
\end{gathered}
$$

where $G$ is a special 2-group of order $2^{6}$ with $Z(G) \cong E_{4}$ in which every maximal subgroup is isomorphic to the minimal non-metacyclic subgroup of order $2^{5}$ (given in (b)).

\section{Proof of Theorem 1.1}

Let $G$ be a non-Dedekindian $p$-group all of whose noncyclic subgroups are normal. In particular, $G$ is nonabelian. If $G$ has no normal elementary abelian subgroup of order $p^{2}$, then $G$ is of maximal class. It follows that $G \cong Q_{16}$ (part (vi) of our theorem).

In what follows we assume that $G$ has a normal abelian subgroup $W$ of type $(p, p)$. Since each subgroup of $G / W$ is normal, $G / W$ is Dedekindian and so $G / W$ is either abelian or Hamiltonian (Proposition 1.2).

Suppose that $p=2$ and there is a normal four-subgroup $W$ such that $G / W$ is Hamiltonian. In that case $\bar{G}=G / W=\bar{Q} \times \bar{A}$, where $\bar{Q}$ is quaternion and $\bar{A}$ is elementary abelian. By Proposition $1.7, d(G) \leq 4$ and so $|\bar{A}| \leq 4$ which implies $2^{5} \leq|G| \leq 2^{7}$.

First we consider the case $|G|=2^{5}$ so that $G=Q$ and $G / W \cong Q_{8}$. Set $S / W=\Phi(G / W)$ so that $S$ is abelian since $\left|G: C_{G}(W)\right| \leq 2$. Suppose that $S$ is elementary abelian. If $s \in S$ is such that $s \notin Z(G)$, then $C_{G}(s) /\langle s\rangle$ contains the four-subgroup $S /\langle s\rangle$, contrary to Proposition 1.7. Hence $S \leq Z(G)$. Since $G$ is not Dedekindian, there is $g \in G-S$ such that $g^{2} \in S-W$ and $\langle g\rangle$ is not normal in $G$. But then $N_{G}(\langle g\rangle)=\langle g\rangle \times W$ and $N_{G}(\langle g\rangle) /\langle g\rangle \cong E_{4}$, contrary to Proposition 1.7. We have proved that $S$ is abelian of type $(4,2)$. If $g \in G-S$, 
then $g^{2} \in S-W$ and so $o(g)=8$. We have proved that $\Omega_{2}(G)=S$ is of order 8. By Proposition 1.4,

$$
G=\left\langle a, b \mid a^{8}=b^{8}=1, a^{4}=b^{4}, a^{b}=a^{-1}\right\rangle
$$

since $M_{2^{n+1}}(n>2)$ does not possess a factor-group isomorphic to $Q_{8}$. We have obtained the group stated in part (v) of our theorem.

Now we consider the case $|G|>2^{5}$. We have $G=Q A, Q \cap A=W$, $Q$ and $A$ are normal in $G, Q / W \cong Q_{8}$, and $A / W$ is elementary abelian of order 2 or 4 . In particular, $\exp (G) \leq 8$. Suppose that $u$ is an involution in $G$ with $u \notin Z(G)$. By Proposition 1.7, $\left|G: C_{G}(u)\right|=2$ and $C_{G}(u) /\langle u\rangle$ is either cyclic of order $\leq 8$ or $C_{G}(u) /\langle u\rangle \cong Q_{8}$. In any case, $|G| \leq 2^{5}$ which is a contradiction. We have shown that $\Omega_{1}(G) \leq Z(G)$ and so $W \leq Z(G)$. Assume that $E=\Omega_{1}(G)$ is of order $\geq 8$. Since $G$ is not Dedekindian, $G$ possesses a cyclic subgroup $Z$ of composite order which is not normal in $G$. Set $\langle z\rangle=Z \cap E$ so that $z$ is a central involution. Let $e, f \in E-\langle z\rangle$ such that $\langle e, f, z\rangle \cong E_{8}$. In this case $S_{1}=\langle Z, e\rangle$ and $S_{2}=\langle Z, f\rangle$ are normal in $G$. But $S_{1} \cap S_{2}=Z$ and so $Z$ is normal in $G$, a contradiction. It follows that $\Omega_{1}(G)=W \leq Z(G)$. Set $T / W=\Phi(Q / W)$ so that $T$ is abelian of type $(4,2)$. For each $x \in Q-T, x^{2} \in T-W$ and so $o(x)=8$ and $\Omega_{2}(Q)=T$ is of order 8 . By Proposition 1.4, $Q$ is a metacyclic group isomorphic to the group of part (v) of our theorem and so $Z(Q) \cong C_{4}$, contrary to the fact that $W \leq Z(G)$.

In what follows we may assume that $G$ has a normal abelian subgroup $W$ of type $(p, p)$ and for each such $W, G / W$ is abelian. This gives that $G^{\prime} \leq W$ and so $G^{\prime}$ is elementary abelian of order $p$ or $p^{2}$. Also, $G$ has no abelian subgroup of type $(p, p, p)$. Indeed, if $E$ is an abelian subgroup of type $(p, p, p)$, then considering maximal subgroups of $E$, we know that they are normal in $G$ and each of them contains $G^{\prime}$ which would imply $G^{\prime}=\{1\}$.

(i) Assume that $Z(G)$ is cyclic. Set $Z=\Omega_{1}(Z(G))$ so that $|Z|=p$ and let $J$ be another subgroup of order $p$. Then $J$ is not normal in $G$ and $W=Z \times J$ is a normal abelian subgroup of type $(p, p)$. If $N=N_{G}(J)$, then $|G: N|=p$ and $N / J \neq\{1\}$ is either cyclic or $p=2$ and $N / J \cong Q_{8}$ (Proposition 1.7).

First suppose that $N / J \cong Q_{8}$ so that $|G|=2^{5}$. We have $W=Z(N)$ and since $G / N$ acts faithfully on $W$, we get $Z=Z(G)$. If $X / J$ is a maximal subgroup of $N / J$, then $X / J \cong C_{4}$ and so $X$ is abelian. Hence $N$ has at least three abelian maximal subgroups which implies $\left|N^{\prime}\right|=2$ and $N^{\prime}<W$ so that $N^{\prime}=Z=Z(G)$. Let $n \in N-W$ so that $n^{2} \in W-J$. Suppose that $n^{2} \notin Z$ so that $\left\langle n^{2}\right\rangle$ is not normal in $G$. By Proposition $1.7,\left\langle n^{2}\right\rangle$ must be a maximal cyclic subgroup in $G$, which is not the case. Thus, $n^{2} \in Z$ and so $\Phi(N)=N^{\prime}=Z$. Let $Q^{*}=\langle a, b\rangle$ be a maximal subgroup of $N$ which does not contain $J=\langle u\rangle$ so that $Q^{*} \cong Q_{8}$ and $N=J \times Q^{*}$. Suppose that there is an element $x \in G-N$ such that $o(x) \leq 4$. Then $x^{2} \in W$ and $D=\langle W, x\rangle \cong D_{8}$. There is $i \in D-N$ such that $i$ is a noncentral involution. By Proposition 1.7, $\left|G: C_{G}(i)\right|=2$ and the fact that $[i, J] \neq\{1\}$ gives that $C_{N}(i)$ covers $N / J$ so 
that $C_{N}(i)=Q_{1} \cong Q_{8}$. We get $G=Q_{1} * D$ is an extraspecial group of order $2^{5}$ and type "-" which appears in part (vii) of our theorem. Suppose that all elements in $G-N$ are of order $>4$ so that $\Omega_{2}(G)=N=J \times Q^{*}$. We use Proposition 1.6 and see that there is an element $y \in G-N$ such that $b^{y}=b u$ and therefore $Q^{*}=\langle a, b\rangle$ is not normal in $G$ in spite of the fact that $Q^{*}$ is noncyclic, a contradiction.

We may assume that $N / J \neq\{1\}$ is cyclic so that $N$ is abelian. Since $J$ is maximal cyclic (Proposition 1.7), $N$ is noncyclic and so $N=J \times A$, where $A$ is cyclic of order $\geq p^{2}$ (since we may assume that $|G|>p^{3}$ ) and so $Z<A$ because $\Omega_{1}\left(\mho_{1}(N)\right)(<A)$ is central in $G$. By Proposition 1.5, $N / Z(G) \cong G^{\prime}$.

Now assume that there exists an element $x$ of order $p$ in $G-N$. Then $\langle Z, x\rangle$ is abelian of type $(p, p)$ so that $\langle Z, x\rangle$ is normal in $G$ and $G^{\prime} \leq W \cap$ $\langle Z, x\rangle=Z$ which implies $G^{\prime}=Z$ and $\left|G^{\prime}\right|=p$. Since $N / Z(G) \cong G^{\prime}$, we have $|N: Z(G)|=p$. But $J \notin Z(G)$ and so we may set $N=Z(G) \times J$, where $Z(G)$ is cyclic. Since $\langle W, x\rangle=G_{0}$ is nonabelian of order $p^{3}$, we get $G=Z(G) * G_{0}$ and this is the case (ii) of our theorem.

Now suppose that there exist no elements of order $p$ in $G-N$ and consider the abelian group $G / W$. If $G / W$ is cyclic, then the fact that $\Omega_{1}(N)=$ $J \times Z=W \cong E_{p^{2}}$ implies that $G$ has a cyclic subgroup of index $p$ and so $G$ is metacyclic. Now, $G^{\prime} \leq W$ and the commutator group of a metacyclic group is cyclic and so $\left|G^{\prime}\right|=p$. By Proposition 1.3, $G$ is minimal nonabelian which gives the case (i) of our theorem. We may assume that $G / W$ is noncyclic and so $G / W$ is abelian of type $\left(p^{a}, p\right)$ with $a \geq 1$. Let $G \geq R>W$ be such that $R / W$ is abelian of type $(p, p)$. Suppose $p=2$ and let $x \in R-N$. Then $x^{2} \in W$ and $[W, x] \neq 1$ so that $\langle W, x\rangle \cong D_{8}$. But then there are involutions in $\langle W, x\rangle-N$, a contradiction. Hence $p>2$. If $R$ (of order $p^{4}$ ) is regular, then $p^{2}=\left|\Omega_{1}(R)\right|=\left|G / \mho_{1}(R)\right|$. Thus, $\mho_{1}(R)=W$ and so there is $x \in R-N$ such that $x^{p} \notin Z$. By Proposition $1.7, x^{p} \in Z(G)$ which implies $W \leq Z(G)$, a contradiction. It follows that $R$ is irregular and so $p=3, R$ is of class 3 and so $R^{\prime}=G^{\prime}=W$. Finally, we know that $N / Z(G) \cong E_{p^{2}}$ (Proposition 1.5) and $R \cap N$ is abelian of type $\left(p^{2}, p\right)$. Thus, if $a \geq 2$, then $R \cap N$ contains a central element of order $p^{2}$, contrary to the fact that $R$ is of maximal class. Hence $a=1, R=G$ and we have obtained the case (iv) of our theorem.

(ii) Assume that $Z(G)$ is noncyclic. Set $Z=Z(G)$ so that $W=\Omega_{1}(Z)=$ $\Omega_{1}(G) \cong E_{p^{2}}$ because $G$ has no elementary abelian subgroup of order $p^{3}$. Moreover, $G^{\prime} \leq W$ so $G$ is of class 2 . For any $x, y \in G,\left[x^{p}, y\right]=[x, y]^{p}=1$ and so $\Phi(G) \leq Z$ and $G / Z$ is elementary abelian.

Suppose that either $p>2$ or $p=2$ and $\left\{x \in G \mid x^{2} \in G^{\prime}\right\} \leq Z$. We show that under this assumption the map $x \rightarrow x^{p}$ is a one-to-one map from $G / W$ into $Z$. Indeed, this is clear for $p>2$ since in that case $x \rightarrow x^{p}(x \in G)$ is a homomorphism with kernel $\Omega_{1}(G)=W$. Let $p=2$. If $x^{2}=y^{2}$, then

$$
\left(x y^{-1}\right)^{2}=x y^{-1} x y^{-1}=x^{2}\left(x^{-1} y^{-1} x y\right) y^{-2}=x^{2}[x, y] y^{-2}=[x, y]
$$


Hence, by our assumption, $x y^{-1} \in Z$ so $x$ and $y$ commute and $\left(x y^{-1}\right)^{2}=$ $x^{2} y^{-2}=1, x y^{-1} \in W=\Omega_{1}(G)$. Thus this fact follows. This gives $|G / W| \leq Z$ and so $|G| \leq|Z||W|$. Since $G$ is nonabelian, we have $G / Z \cong E_{p^{2}}$ and the map $x \rightarrow x^{p}$ is onto which implies $Z=\Phi(G)$. Each maximal subgroup of $G$ is abelian and so $G$ is minimal nonabelian. Since $\Omega_{1}(G)=W \cong E_{p^{2}}$, Proposition 1.3 shows that $G$ is also metacyclic and we have obtained the groups from part (i) of our theorem.

In the rest of the proof we may assume that $p=2$ and $\left\{x \in G \mid x^{2} \in G^{\prime}\right\} \not{Z}$ $Z$. Hence there is an element $x \in G-Z$ with $x^{2} \in G^{\prime} \leq W$ and $o(x)=4$. Suppose there is $y \in Z$ with $y^{2}=x^{2}$. Then $\left(x y^{-1}\right)^{2}=1$ so $x y^{-1} \in W \leq Z$ and $x \in Z$, a contradiction. This implies that $Z=Z_{1} \times\left\langle x^{2}\right\rangle$, where $Z_{1}$ is cyclic. Since $\Omega_{2}(G) \not \leq Z(G)$, Proposition 1.8 implies that $G$ has a non-normal cyclic subgroup $H$ of order 4. By Proposition 1.7, $H$ is maximal cyclic in $G$, $|G: N|=2$ with $N=N_{G}(H)$ and $N / H$ is either cyclic or quaternion.

First suppose $N / H \cong Q_{8}$ so that $|G|=2^{6}$. Set $S / H=\Phi(N / H)$ so that $S$ is abelian of type $(4,2)$ because $\left|N: C_{N}(H)\right| \leq 2$ and $H$ is maximal cyclic. We have $\Omega_{1}(S) \cong E_{4}$ which implies that $\Omega_{1}(S)=W=\Omega_{1}(G) \leq Z(G)$. On the other hand, $Z(G) \leq N$ and since $H \not \leq Z(G)$, we get $W=Z(G)$. Since $G / Z(G)$ is elementary abelian and $d(G) \leq 4$, we have $\Phi(G)=W$ and $\exp (G)=4$.

Suppose in addition that $G$ possesses a subgroup $Q=\langle k, l\rangle \cong Q_{8}$ so that $Q$ is normal in $G$. Since $G^{\prime} \leq W$, no element $y$ in $G$ induces an outer automorphism on $Q$ (otherwise, we may assume that $k^{y}=l$ and then $[k, y]=$ $k^{-1} l$, where $\left.o\left(k^{-1} l\right)=4\right)$. It follows that $L=C_{G}(Q)$ covers $G / Q$ (noting that $\left.\operatorname{Aut}\left(Q_{8}\right) \cong S_{4}\right)$ and so $G=Q * L$ with $Q \cap L=Z(Q)=\langle z\rangle$ and $k^{2}=l^{2}=z$. We have $W=Z(G) \leq L$ and since $Z(L) \leq Z(G)$, we get $Z(L)=\Omega_{1}(L)=W$. Also, $\Phi(G)=\Phi(Q) \Phi(L)$ which gives $\Phi(L)=W$. The subgroup $L$ is obviously minimal nonabelian (of exponent 4) and $L$ is metacyclic since $\Omega_{1}(L) \cong E_{4}$ (Proposition 1.3). We get

$$
L=\left\langle a, b \mid a^{4}=b^{4}=1, a^{b}=a^{-1}\right\rangle,
$$

where $W=Z(L)=\left\langle a^{2}, b^{2}\right\rangle$ and $a^{2}$ and $b^{2}$ are the only involutions in $W$ which are squares in $L$. If $c \in L$ is such that $c^{2}=z$, then $o(k c)=2$ and $k c \notin L$, a contradiction. It follows that $a^{2} \neq z$ and $b^{2} \neq z$ which implies $a^{2} b^{2}=z$. We have $\langle b k, a\rangle=R \cong Q_{8}$ since $(b k)^{2}=b^{2} k^{2}=b^{2} z=a^{2}$ and $(b k)^{a}=b a^{2} k=(b k) a^{2}=(b k)^{-1}$. Also, $R \cap Q=\{1\}$ and $R$ is normal in $G$ so that $G=Q \times R$. On the other hand, $(b k)^{l}=(b k) z$ and so $z \in R$, a contradiction. We have proved that a quaternion group is not a subgroup of $G$.

Let $X$ be any minimal nonabelian subgroup of $G$. Since $X$ is not isomorphic to $Q_{8},\left|\Omega_{1}(X)\right| \leq 4$, and $\exp (X)=4$, Proposition 1.3 implies that $X$ is isomorphic to the group

$$
H_{2}=\left\langle a, b \mid a^{4}=b^{4}=1, a^{b}=a^{-1}\right\rangle .
$$


By Proposition 1.9, $G$ is the special group given in part (ix) of our theorem.

In what follows we assume that $N / H$ is cyclic which implies $d(G) \leq 3$.

(ii1) Suppose $\left|G^{\prime}\right|=2$. Set $H=\langle h\rangle \cong C_{4}$ and let $N \geq K>H$ be such that $|K / H|=2$. Then $K$ is normal in $G$ (since $K$ is noncyclic) and if $g \in G-N$, then $h^{g}=h i$ with an involution $i \in K-H$ and $i \in G^{\prime}$ so that $G^{\prime}=\langle i\rangle$. In that case $H \leq Z(N)$ which gives that $N$ is abelian. By Proposition $1.5, N / Z \cong G^{\prime}$ and so $G / Z \cong E_{4}$. We know that there is an element $x \in G-Z$ such that $x^{2}=i$ and so $\langle x\rangle$ is normal in $G$. Now, $Z_{1} \cap\langle x\rangle=\{1\}$ and so $\left(Z_{1} \times\langle x\rangle\right) /\langle x\rangle$ has index 2 in $G /\langle x\rangle$. If $G /\langle x\rangle$ is cyclic, then $G$ is metacyclic which together with $\left|G^{\prime}\right|=2$ implies that $G$ is minimal nonabelian (Proposition 1.3) and we have obtained groups of part (i) of our theorem. If $G /\langle x\rangle$ is noncyclic, then $G /\langle x\rangle$ is abelian of type $\left(2^{n}, 2\right), n \geq 1$ (since we may assume $|G| \geq 2^{4}$ ), and so there is $y \in G-\left(\langle x\rangle \times Z_{1}\right.$ ) such that $y^{2} \in\langle x\rangle$. But $\langle x\rangle \not Z Z(G)$ and so $y^{2} \in\langle i\rangle$ which together with $x^{y}=x^{-1}$ implies that $\langle x, y\rangle \cong Q_{8}$ and so $G=\langle x, y\rangle \times Z_{1}$ with $\left|Z_{1}\right|>2$. We have obtained the groups stated in part (iii) of our theorem.

(ii2) Now suppose $\left|G^{\prime}\right|=4$ so that $G^{\prime}=W=\Omega_{1}(Z)=\Omega_{1}(G)$. By Proposition $1.5,|G / Z|=8$ and so $G / Z \cong E_{8}$ which implies $d(G)=3$ and so $Z=\Phi(G)$.

Suppose that $G$ possesses a subgroup $\langle x, y\rangle=Q \cong Q_{8}$, where we set $Z(Q)=\langle z\rangle$. Then $Q$ is normal in $G$ and again there is no element in $G$ inducing an outer automorphism on $Q$ (since $G^{\prime} \cong E_{4}$ ). It follows that $G=$ $Q C_{G}(Q)$ with $Q \cap C_{G}(Q)=\langle z\rangle$. We get $\Phi(G) \geq\langle z\rangle$ and $\Phi(G) \leq C=C_{G}(Q)$ and so $|C: \Phi(G)|=2$. Since $Z=\Phi(G), C$ is abelian and so $G^{\prime}=\langle z\rangle$, a contradiction. Hence $Q_{8}$ is not a subgroup of $G$.

Assume that $Z>W=G^{\prime}$ and so $G / W$ is abelian of type $\left(2^{m}, 2,2,\right)$, $m>1$. Set $M / W=\Omega_{1}(G / W)$ so that $M / W \cong E_{8}$. Set $Z_{0}=M \cap Z$, where $\left|Z_{0}: W\right|=2$ and $Z_{0}$ is abelian of type $(4,2)$. Let $v \in Z_{0}-W$ so that $o(v)=4$ and $v^{2}=u \in W$. Let $R / W$ be a complement of $Z_{0} / W$ in $M / W$. We have $|R|=2^{4}$ and $R / W \cong E_{4}$. Suppose there is $y \in R$ such that $y^{2}=u$. Then $o(y v)=2$ and $y v \notin R$, a contradiction. It follows that $R$ is nonabelian (otherwise, $R \cong C_{4} \times C_{4}$ and in that case $u$ is a square in $R$ ) and since $Q_{8}$ is not a subgroup of $R$, we get that $R$ is minimal nonabelian with $\Omega_{1}(R)=W$ and $\exp (R)=4$. By Proposition 1.3,

$$
R=\left\langle a, b \mid a^{4}=b^{4}=1, a^{b}=a^{-1}\right\rangle=H_{2}
$$

and we must have $a^{2} \neq u$ and $b^{2} \neq u$ so that $a^{2} b^{2}=u$. Then we get

$$
(b v)^{2}=b^{2} v^{2}=b^{2} u=a^{2} \text { and } a^{b v}=a^{-1}
$$

so that $\langle a, b v\rangle \cong Q_{8}$, a contradiction.

We have proved that $Z=W$ and so $G^{\prime}=W=Z(G)=\Phi(G)=\Omega_{1}(G) \cong$ $E_{4}$ and so $G$ is a special group of order $2^{5}$. Since $Q_{8}$ is not a subgroup of $G$ and $\Omega_{1}(G) \cong E_{4}$, each minimal nonabelian subgroup of $\mathrm{G}$ is isomorphic to the 
above group $H_{2}$. By Proposition 1.9, $G$ is the minimal non-metacyclic group of order $2^{5}$ and so we have obtained the group of part (viii) of our theorem.

It is an easy exercise to show that all the groups from Theorem 1.1 satisfy the assumptions of this theorem and we are done.

\section{REFERENCES}

[1] Y. Berkovich, Groups of prime power order, I and II (with Z. Janko), in preparation.

[2] Y. Berkovich and Z. Janko, Structure of finite p-groups with given subgroups, Contemp. Math. 402 (2006), 13-93.

[3] B. Huppert, Endliche Gruppen I, Die Grundlehren der Mathematischen Wissenschaften 134, Springer-Verlag, Berlin-New York, 1967.

[4] Z. Janko, Finite 2-groups with exactly four cyclic subgroups of order $2^{n}$, J. Reine Angew. Math. 566 (2004), 135-181.

[5] Z. Janko, On finite nonabelian 2-groups all of whose minimal nonabelian subgroups are of exponent 4, J. Algebra 315 (2007), 801-808.

[6] D. S. Passman, Nonnormal subgroups of p-groups, J. Algebra 15 (1970), 352-370.

\section{Z. Božikov}

Faculty of Civil Engineering and Architecture

University of Split

21000 Split

Croatia

E-mail: Zdravka.Bozikov@gradst.hr

Z. Janko

Mathematical Institute

University of Heidelberg

69120 Heidelberg

Germany

Received: 4.3.2008.

Revised: 15.4.2008. 